\title{
RECONSTRUCTING THE AXIAL VOID VELOCITY PROFILE IN BWRS FROM MEASUREMENTS OF THE IN-CORE NEUTRON NOISE
}

\author{
Imre Pázsit ${ }^{1}$, Mathieu Hursin ${ }^{2,3}$, Henrik Nylén $^{4}$ and Victor Dykin ${ }^{1}$ \\ ${ }^{1}$ Chalmers University of Technology, Division of Subatomic and Plasma Physics, \\ Nuclear Engineering Group, \\ SE-412 96 Gteborg, Sweden \\ ${ }^{2}$ Paul Scherrer Institut, Nukleare Energie und Sicherheit, \\ PSI Villigen 5232, Switzerland \\ ${ }^{3}$ Ecole Polytechnique Fédérale de Lausanne (EPFL), \\ Switzerland \\ ${ }^{4}$ Ringhals $\mathrm{AB}$, \\ SE-432 85 Väröbacka, Sweden
}

imre@chalmers.se, mathieu.hursin@epfl.ch, Henrik.Nylen@vattenfall.com, victor@ nephy.chalmers.se

\begin{abstract}
The problem of determining the axial velocity profile from the in-core neutron noise measurements is revisited, with the purpose of developing an objective method for the determination of the void fraction. Until now it was assumed that in order to determine a realistic velocity profile which shows an inflection point and hence has to be at least a third order polynomial, one needs four transit times and hence five in-core detectors at various axial elevations. However, attempts to determine a fourth transit time by adding a TIP detector to the existing four LPRMs and cross-correlate it with any of the LPRMs were unsuccessful so far. In this paper we thus propose another approach, where the TIP detector is only used for the determination of the axial position of the onset of boiling. By this approach it is sufficient to use only three transit times. Moreover, with another parametrisation of the velocity profile, it is possible to reconstruct the velocity profile even without knowing the onset point of boiling, in which case the TIP is not needed. In the paper the principles are explained and the strategy is demonstrated by concrete examples.
\end{abstract}

KEYWORDS: BWR; void velocity profile; neutron noise; transit time

\section{INTRODUCTION}

Determination of the local void content in BWRs from measurements with existing instrumentation has been a matter of interest for a long time. Although several suggestions were put forward concerning how to extract the local void fraction from in-core neutron noise measurements, the 
suggested methods were incomplete and required either calibration, or several auxiliary conditions, whose fulfilment was unclear and rather uncertain, or both.

Recently, a method was suggested by some of the present authors to overcome the deficiency of the above mentioned methods, which is capable to determine the local void content in an absolute way, without the need of calibrations, or basic assumptions on the void profile [1]. The method is primarily based on the recognition that it is necessary to know the local void velocity at the neutron detector positions. However, in measurements only the transit times of the void between two axially displaced neutron detectors can be obtained. The transit times are integrals of the inverse velocity, which is not constant between the detectors. The relationship between the void velocity at the detector positions, and the transit time between the detector pairs, is rather involved and implicit, and to some extent underdetermined, as it will be elaborated on below.

Usually, the standard instrumentation of BWRs comprises 4 detectors axially at one radial core position, hence one has access to three transit times between the three detector pairs, The only possibility to determine the velocity at the detector positions is to reconstruct the whole axial velocity profile. Since the axial depencence of the velocity has an inflection point, it has to be described by a non-linear function. The simplest such function, which was also suggested in [1] and [2], and which is the only one tested so far, is a third order polynomial. However, it turns out that this choice is neither justified, nor optimal, and moreover its application led to difficulties, as described in the above mentioned publications.

One difficulty is that a third order polynomial has four parameters. To determine these, one would need four independent transit times, hence access to five detectors. To solve this problem, it was suggested that one could use, in addition to the four standard LPRMs, a TIP detector, and make a measurement at an axial position either between the four LPRMs, or outside these, i.e. in a position different from those of the LPRM positions. This approach was tried in measurements, performed in the Swedish Ringhals-1 BWR [3]. Unfortunately, as is also described in [3], the attempt was unsuccessful. Due to the fact that the data acquisition for the LPRMs and the TIP detectors is made by different equipment with different buffering properties, the data acquisition for them was not completely synchronouos. There is the further difficulty of the inaccuracy of the positioning of the TIP detectors. The conclusion in [3] was that the application of the TIP detector for acquiring a fourth transit time is not feasible.

In this paper therefore we suggest a different strategy. First, we realise that there is no need for a fourth transit time to determine four parameters of velocity profile, either a polynomial or some other form, if the axial point of the onset of the boiling is known. The onset point of the boiling can be determined with a TIP detector alone, from the amplitude of its root mean square noise (RMS) or its APDS, or, alternatively, from the coherence between the TIP and the lowermost LPRM, if these are determined as a function of the axial position of the TIP. At the onset of the boiling the void velocity can be assumed to be equal to the inlet coolant velocity, which is known, Thus, knowledge of these two quantities reduces therefore the number of unknowns of the axial velocity profile to be determined from four to three.

Second, there are many non-linear functions with an inflection point, which represent a much higher order non-linearity than third order, but which nevertheless can be parametrised with only three parameters instead of four. In this case not even the onset point of the boiling needs to be known; determination of the void profile is then possible based on solely of the three measured 
transit times with the standard instrumentation, without the need for using a TIP detector at all. In this paper this latter approach will be developed and illustrated by examples.

\section{THE VELOCITY PROFILE AND ITS MODELLING}

\subsection{Characteristics of the Velocity Profile}

As is general in reactor noise diagnostics problems, when only a limited number of measurements is available, obtained from detectors in a few specified spatial positions, this is not sufficient to reconstruct the full spatial dependence of the noise source. Inevitably, one needs to make an assumption on the space dependence of the noise source in an analytical form, which contains only a limited number of free parameters. These can then be determined from the limited number of measurements [4].

While this strategy is easy to follow for localised perturbations, since these can be simplified to a spatial Dirac-delta function, for the velocity profile the case is more complicated. A further difficulty is that, for obvious reasons, no directly measured velocity profiles are available. Only qualitative information is known either from common sense considerations, or from simulations. A few profiles are shown below, taken either from calculations by system codes (TRACE), Fig. 1, or from simulations of a bubbly flow in a heated channel, by an in-house Monte Carlo code [2], Fig. 2. In the TRACE calculations, account was taken for the fact that the boiling does not start at the inlet, rather at a higher elevation.

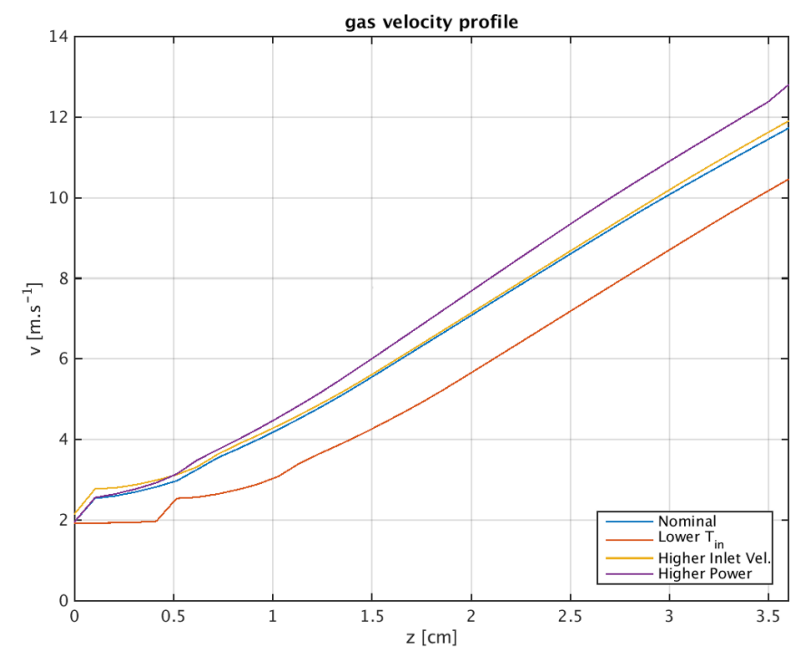

Figure 1: Void velocity profiles simulated by TRACE

What these figures tell us is that the velocity increases monotonically in the channel from the inlet, first at least quadratically, then the increase slows down, either leading to an inflection point, or to a linear increase towards the core exit. Apparently, to find out the best analytical form of the axial velocity profile, a much wider data base should be available for each different reactor construction. 

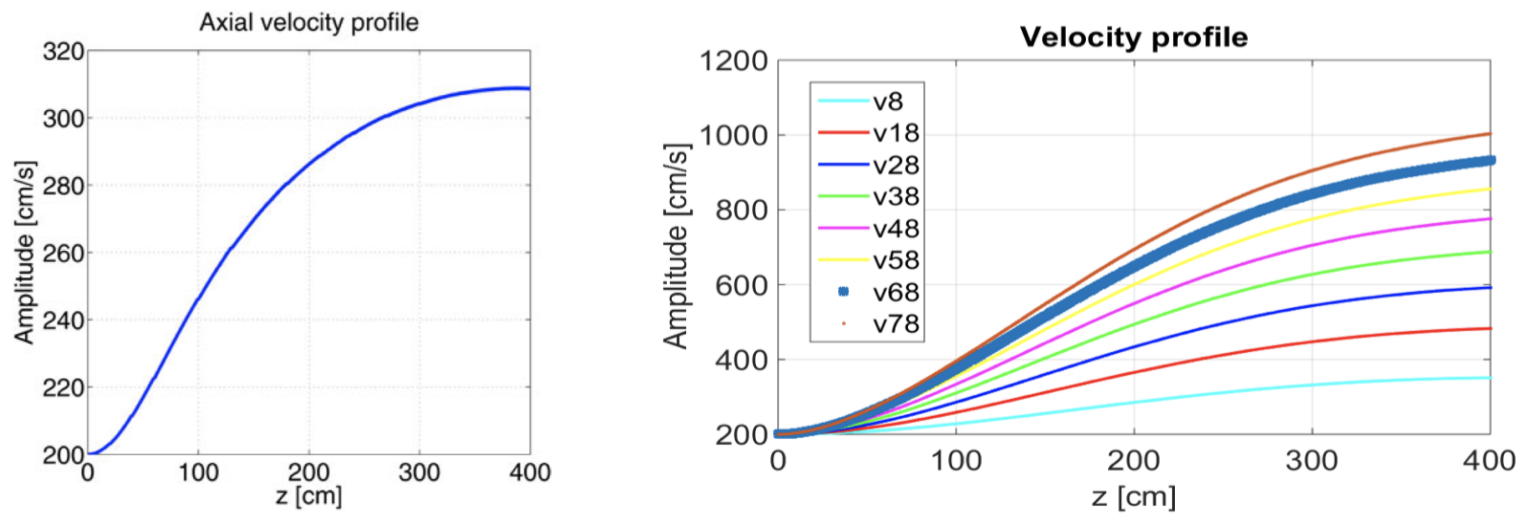

Figure 2: Void velocity profiles simulated by a Monte-Carlo model of bubbly two-phase flow

\subsection{Possible Analytical Forms}

In the previous works [1-3] a third order polynomial was assumed:

$$
v(z)=a+b z+c z^{2}+d z^{3}
$$

This form has a few drawbacks, namely that a) the integral of $v^{-1}(z)$ w.r.t. $z$ does not exist in an analytical form, and $\mathrm{b}$ ) that it assumes that the boiling starts at the inlet, i.e. at $z=0$, which is not true in practical cases. Accounting for the fact that the onset of the boiling is at $z=h$ where $h$ is an unknown, would increase the number of parameters to be determined to 5. On the other hand, the property a) does not represent a significant difficulty, since the unknown parameters $a-d$ can also be determined by numerical unfolding methods, as it will be shown below.

If, as suggested in this work, the onset point $z=h$ of the boiling is known from measurements, then the third order polynomial form of (1) can be written in the form

$$
v(z)=\Delta(z-h)\left[v_{0}+b(z-h)+c(z-h)^{2}+d(z-h)^{3}\right]
$$

where $\Delta(z)$ is the unit step function, and $v_{0}$ is the (known) inlet coolant velocity. This form contains only three unknowns, which can be determined from the three measured transit times.

It is easy to see that there are other non-linear functions with an inflection point which require even fewer unknown parameters. These include trigonometric functions, such as $a \cdot \operatorname{atan}(b(z-c))$, where $a, b$ and $c$ are constants, or the co-called "sigmoid" function, used in the training of ANNs. In such a case the onset point of the boiling does not need to be known. In the next section such a model is proposed, and a procedure for its use for the unfolding of the velocity profile is suggested.

\section{CONSTRUCTION OF A SIMPLE VELOCITY PROFILE}

In order to obtain a velocity profile with an inflection point, which can be described by a few parameters, we shall assume a very simple phenomenological model based on simple considerations. The model does not have any deep physical meaning, it just makes is simpler to estimate the possible range of the model parameters (which is useful in the inversion process). 
Assume that the core boundaries lie between $z=0$ and $z=H$ with a static flux $\phi(z)$. Assuming that the boiling starts at the axial elevation $z=h$, and that there is a simple monotonic relationship between void fraction and void velocity, and that the latter at point $z$ is proportional to the accumulated heat production between the boiling onset and the actual position, gives the form

$$
v(z)=\Delta(z-h)\left\{v_{0}+c \int_{h}^{z} \phi(z) d z\right\} .
$$

where $h$ and $c$ are unknown constants *. Assume now, for simplicity, a simple flux shape as

$$
\phi(z)=\cos [B(z-H / 2)]
$$

Here, it is not assumed that $B=\pi / H$, rather $B$ is kept as an independent (unknown) parameter. By allowing $B<\pi / H$, an extrapolation length, as an independent parameter, can be accounted for.

With this choice, after integration, the velocity profile is obtained in the simple form

$$
v(z)=\Delta(z-h)\left\{a_{1}+c_{1} \sin \left[B\left(z-\frac{H}{2}\right)\right]\right\}
$$

with

$$
a_{1}=v_{0}-\frac{c}{B} \sin \left[B\left(h-\frac{H}{2}\right)\right] \quad \text { and } \quad c_{1}=\frac{c}{B}
$$

Before turning to the problem of recovering the velocity profile from the measured transit times, a qualitative illustration will be given. To this order, geometrical and velocity data will be taken from the Ringhals-1 plant. The geometrical arrangement is depicted on Fig. 3, giving the core height and the axial positions of the detectors from the core bottom. The inlet coolant velocity is $v_{\text {in }}=v_{0}=2 \mathrm{~m} / \mathrm{s}$, and the outlet void velocity is about $12 \mathrm{~m} / \mathrm{s}$. Assuming an extrapolation distance of $0.2 \mathrm{~m}$ for the flux, and assuming the onset of the boiling at $h=0.2 \mathrm{~m}$, the static flux and the arising velocity profile are shown in Fig. 4.

\section{THE UNFOLDING PROCEDURE}

\subsection{Unfolding Strategies}

The velocity profile given in Eq. (5) depends only on three parameters, hence three transit times, derived from four LPRM signals, should be sufficient for reconstucting the velocity profile. Eq. (5) has the further property that its inverse is analytically integrable, thereby giving a possibility to express the transit time $t_{1,2}$ of the void between the detector positions $z_{1}$ and $z_{2}$, with $z_{1}<z_{2}$, as analytical functions of the unknown parameters $a_{1}, c_{1}$ and $B$. For practical reasons we will number the detector positions such that $z_{1}$ corresponds to the lowermost detector, LPRM 4, and the transit times between the detector pairs will be indexed by the position of the lower detector, i.e. $t_{1,2} \equiv t_{1}$ etc. With these notations, one has

$$
t_{i}\left(a_{1}, c_{1}, B\right)=\int_{z_{i}}^{z_{i+1}} \frac{d z}{v(z)}=
$$

${ }^{*}$ As mentioned earlier, if needed, $h$ can be determined by measurements with a TIP detector. 


$$
\frac{2\left(\tan ^{-1}\left(\frac{c_{1}-a_{1} \tan \left(\frac{1}{4} B\left(H-2 z_{i+1}\right)\right)}{\sqrt{a_{1}^{2}-c_{1}^{2}}}\right)-\tan ^{-1}\left(\frac{c_{1}-a_{1} \tan \left(\frac{1}{4} B\left(H-2 z_{i}\right)\right)}{\sqrt{a_{1}^{2}-c_{1}^{2}}}\right)\right)}{B \sqrt{a_{1}^{2}-c_{1}^{2}}}
$$

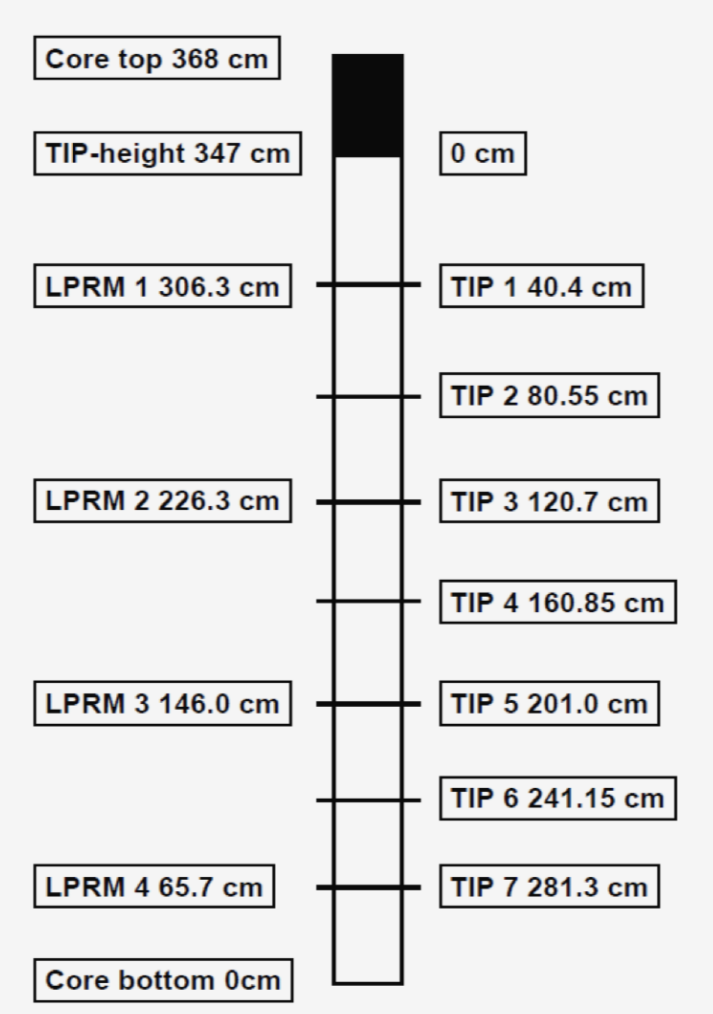

Figure 3: Layout of the measurements
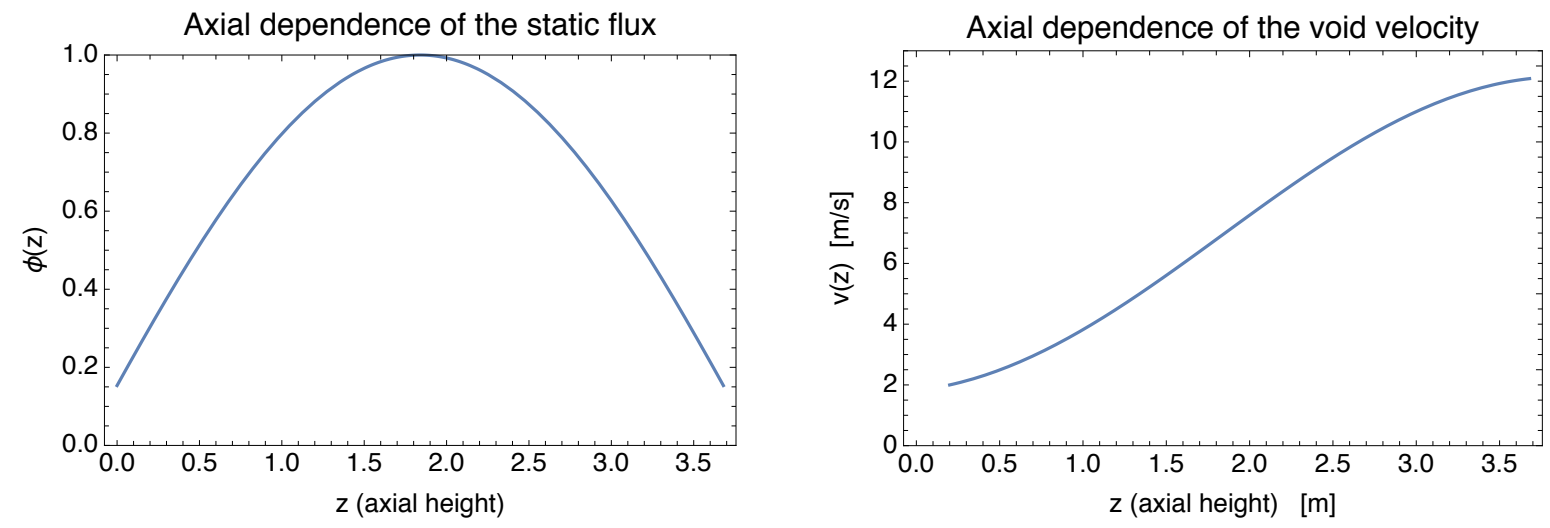

Figure 4: Flux and void velocity profile 
Our expectation was that in possession of the analytical expressions for $t_{i}\left(a_{1}, c_{1}, B\right), i=1,2,3$ in the above form, and having access to given values of the three measured transit times $\tau_{i}, i=1,2,3$, the unknown parameters $a_{1}, c_{1}, B$ can be determined as the roots of the non-linear equation system

$$
t_{i}\left(a_{1}, c_{1}, B\right)=\tau_{i}, \quad i=1,2,3
$$

This strategy was tested by choosing detector positions, core size, as well as inlet coolant velocity and the same value for $c_{1}$ which were used in calculating the profile in the right hand side of Fig. 4. Having the analytical form of $v(z)$, the concrete transit times $\tau_{i}, i=1,2,3$ can be numerically evaluated and used in (8), with the $t_{i}$ given in the analytical form (7). For the numerical solution of this non-linear equation system, the numerical root finding routine NSolve of Mathematica 12.0.0.0 was used [5]. However, the root finding did not converge, even if quite accurate starting values were specified. It appears that the NSolve routine is primarily designed for treating polynomial equations, rather than transcendental ones.

Therefore, another path was followed to unfold the parameters of the void profile from the transit times. Instead of using Nsolve, a kind of fitting procedure was selected by searching for the minimum of the penalty function

$$
\sum_{i}^{3}\left[t_{i}\left(a_{1}, c_{1}, B\right)-\tau_{i}\right]^{2}
$$

as functions of $a_{1}, c_{1}$ and $B$. The FindMinimum routine of Mathematica 12.0.0.0. was used. This procedure worked well and was able to reproduce the input parameters of the velocity profile. Initially the analytical form (7) was used for the $t_{i}\left(a_{1}, c_{1}, B\right)$. However, it turned out that defining these latter as numerical integrals with free parameters $a_{1}, c_{1}, B$ worked much faster and better precision, showing also that for the unfolding, it is not necessary that the transit times are given in an analytical form. Consequently, the modified polynomial form of $v(z)$ in Eq. (2) can also be used, despite that $v^{-1}(z)$ is not integrable analytically.

The procedure was tested using the velocity profile given in (5) with various values of the parameters, also with combinations that yielded velocity profiles similar to those in Fig. 1. In all cases the procedure worked correctly and accurately.

\subsection{Test with Ringhals-1 Data}

It might be interesting to test the procedure with pure measurement data, where the true values of the flow profile parameters are not known. This has the disadvantage, that in such a case the validity of the reconstructed velocity profile cannot be verified, but it is a test of whether the unfolding procedure works when one cannot give a qualified guess of the starting values for the search of the minimum. To this end we took real measurement data from Ringhals-1 [3]. These are given below in Table 1.

The search for the parameter values which give the minimum of (9) yielded feasible values for the parameters $a_{1}, c_{1}$ and $B$. The corresponding velocity profile is shown in Fig. 5, not surprisingly showing a similarity with the previous model profile. As noted, the success of the procedure is not a proof of the accuracy and correctness of the unfolding procedure, but it gives a good start in searching for realistic velocity profiles and stable unfolding procedures. 
Table 1: Transit times from Ringhals-1 (from [3])

\begin{tabular}{|c|c|c|}
\hline$\tau_{1}[\mathrm{~s}]$ & $\tau_{2}[\mathrm{~s}]$ & $\tau_{3}[\mathrm{~s}]$ \\
\hline 0.2712 & 0.2111 & 0.1253 \\
\hline
\end{tabular}

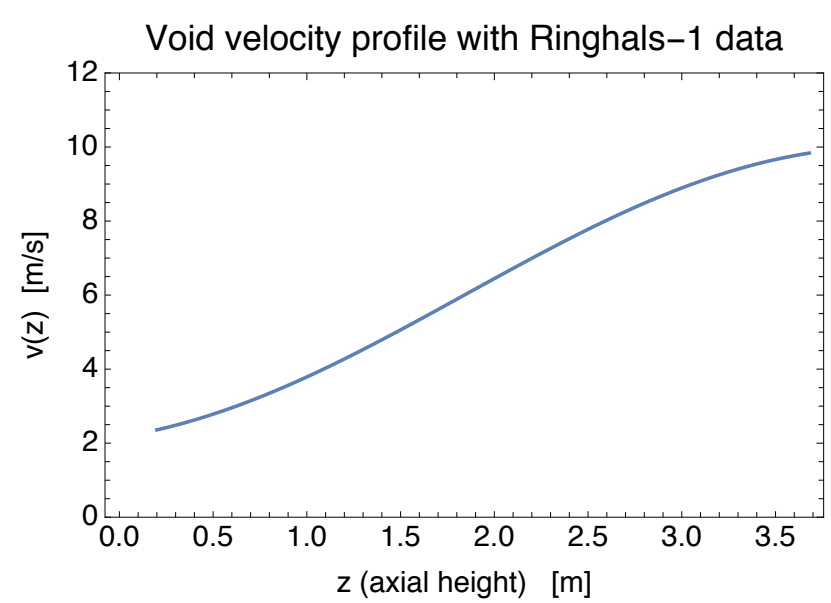

Figure 5: Void velocity profile obtained from Ringhals measurements

\section{CONCLUSIONS}

Further work is required in several areas. First, high-fidelity realistic void velocity profiles need to be generated by system codes, and the best functional form of the profile should be established. Also, the model introduced in this work could be easily extended to depend on four parameters, such that it can describe more complicated profiles than those shown here.

\section{REFERENCES}

[1] V. Dykin and I. Pázsit. "Simulation of in-Core Neutron Noise Measurements for Axial Void Profile Reconstruction in Boiling Water Reactors." Nuclear Technology, volume 183(3), pp. 354-366 (2013).

[2] I. Pázsit, C. Montalvo Martín, V. Dykin, and H. Nylén. "Final Report on the Research Project Ringhals Diagnostics and Monitoring, Stage 14." CTH-NT-253/RR-16, Chalmers University of Technology, Göteborg, Sweden (2011).

[3] V. Dykin, C. Montalvo Martín, H. Nylén, and I. Pázsit. "Ringhals Diagnostics and Monitoring, Final Research Report 2012 - 2014." CTH-NT-304/RR-19, Chalmers University of Technology, Göteborg, Sweden (2014).

[4] I. Pázsit and C. Demazière. Noise Techniques in Nuclear Systems, volume 2 of Handbook of Nuclear Engineering, pp. 1631 - 1737. Springer Science (2010).

[5] "Wolfram Research, Inc., Mathematica, Version 12.0.0.0.” Champaing, IL. (2019). 